Acta Crystallographica Section E

Structure Reports

Online

ISSN 1600-5368

\section{5-Cyclohexyl-2-(3-fluorophenyl)-3- methylsulfinyl-1-benzofuran}

\section{Hong Dae Choi, ${ }^{a}$ Pil Ja Seo ${ }^{a}$ and Uk Lee ${ }^{b *}$}

a Department of Chemistry, Dongeui University, San 24 Kaya-dong Busanjin-gu, Busan 614-714, Republic of Korea, and ${ }^{\mathbf{b}}$ Department of Chemistry, Pukyong

National University, 599-1 Daeyeon 3-dong, Nam-gu, Busan 608-737, Republic of Korea

Correspondence e-mail: uklee@pknu.ac.kr

Received 20 February 2012; accepted 24 February 2012

Key indicators: single-crystal X-ray study; $T=173 \mathrm{~K}$; mean $\sigma(\mathrm{C}-\mathrm{C})=0.003 \AA$; $R$ factor $=0.041 ; w R$ factor $=0.096 ;$ data-to-parameter ratio $=19.8$.

In the title compound, $\mathrm{C}_{21} \mathrm{H}_{21} \mathrm{FO}_{2} \mathrm{~S}$, the cyclohexyl ring adopts a chair conformation. The 3-fluorophenyl ring makes a dihedral angle of $38.38(6)^{\circ}$ with the mean plane [r.m.s. deviation $=0.010$ (1) $\AA$ ] of the benzofuran fragment. In the crystal, molecules are linked by weak $\mathrm{C}-\mathrm{H} \cdots \mathrm{O}$ hydrogen bonds.

\section{Related literature}

For background information and the crystal structures of related compounds, see: Choi et al. $(2011 a, b)$.

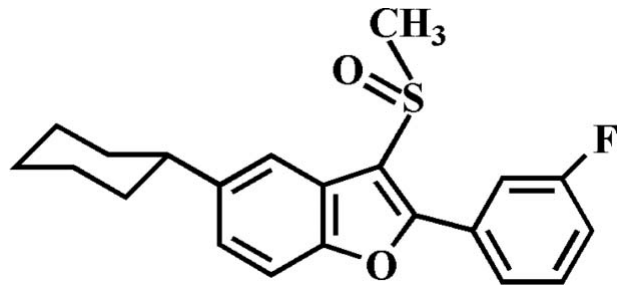

\section{Experimental}

Crystal data

$\mathrm{C}_{21} \mathrm{H}_{21} \mathrm{FO}_{2} \mathrm{~S}$

$M_{r}=356.44$
Orthorhombic, Pna2 $a=12.7767$ (10) §

$$
\begin{aligned}
& b=13.0764(10) \AA \\
& c=10.7711(8) \AA \\
& V=1799.6(2) \AA^{3} \\
& Z=4
\end{aligned}
$$

$$
\begin{aligned}
& \text { Mo } K \alpha \text { radiation } \\
& \mu=0.20 \mathrm{~mm}^{-1} \\
& T=173 \mathrm{~K} \\
& 0.34 \times 0.24 \times 0.20 \mathrm{~mm}
\end{aligned}
$$

\section{Data collection}

Bruker SMART APEXII CCD diffractometer

Absorption correction: multi-scan (SADABS; Bruker, 2009)

$T_{\min }=0.935, T_{\max }=0.961$

Refinement

$R\left[F^{2}>2 \sigma\left(F^{2}\right)\right]=0.041$

$w R\left(F^{2}\right)=0.096$

$S=1.03$

4503 reflections

227 parameters

1 restraint
17893 measured reflections 4503 independent reflections 4036 reflections with $I>2 \sigma(I)$ $R_{\text {int }}=0.058$

Table 1

Hydrogen-bond geometry $\left(\AA{ }^{\circ}\right)$.

\begin{tabular}{lllll}
\hline$D-\mathrm{H} \cdots A$ & $D-\mathrm{H}$ & $\mathrm{H} \cdots A$ & $D \cdots A$ & $D-\mathrm{H} \cdots A$ \\
\hline $\mathrm{C} 21-\mathrm{H} 21 B \cdots \mathrm{O} 2^{\mathrm{i}}$ & 0.98 & 2.30 & $3.276(3)$ & 176 \\
\hline Symmetry code: $(\mathrm{i})-x+1,-y, z-\frac{1}{2}$. & &
\end{tabular}

Data collection: APEX2 (Bruker, 2009); cell refinement: SAINT (Bruker, 2009); data reduction: $S A I N T$; $\operatorname{program}(\mathrm{s})$ used to solve structure: SHELXS97 (Sheldrick, 2008); program(s) used to refine structure: SHELXL97 (Sheldrick, 2008); molecular graphics: ORTEP-3 (Farrugia, 1997) and DIAMOND (Brandenburg, 1998); software used to prepare material for publication: SHELXL97.

This work was supported by a Dongeui University Foundation grant (2011).

Supplementary data and figures for this paper are available from the IUCr electronic archives (Reference: BH2416).

\title{
References
}

Brandenburg, K. (1998). DIAMOND. Crystal Impact GbR, Bonn, Germany. Bruker (2009). APEX2, SADABS and SAINT. Bruker AXS Inc., Madison, Wisconsin, USA.

Choi, H. D., Seo, P. J., Son, B. W. \& Lee, U. (2011a). Acta Cryst. E67, o281. Choi, H. D., Seo, P. J., Son, B. W. \& Lee, U. (2011b). Acta Cryst. E67, o470.

Farrugia, L. J. (1997). J. Appl. Cryst. 30, 565.

Flack, H. D. (1983). Acta Cryst. A39, 876-881.

Sheldrick, G. M. (2008). Acta Cryst. A64, 112-122. 


\section{supporting information}

Acta Cryst. (2012). E68, o944 [https://doi.org/10.1107/S1600536812008343]

\section{5-Cyclohexyl-2-(3-fluorophenyl)-3-methylsulfinyl-1-benzofuran}

\section{Hong Dae Choi, Pil Ja Seo and Uk Lee}

\section{S1. Comment}

As a part of our ongoing study of 5-cyclohexyl-3-methylsulfinyl-1-benzofuran derivatives containing either 2-phenyl (Choi et al., 2011a) or 2-(4-fluorophenyl) (Choi et al., 2011b) substituents, we report herein the crystal structure of the title compound.

In the title molecule (Fig. 1), the benzofuran unit is essentially planar, with a mean deviation of 0.010 (1) $\AA$ from the least-squares plane defined by the nine constituent atoms. The cyclohexyl ring is in the chair form. The dihedral angle between the 3 -fluorophenyl ring and the mean plane of the benzofurn fragment is $38.38(6)^{\circ}$. The crystal packing is stabilized by weak intermolecular $\mathrm{C}-\mathrm{H} \cdots \mathrm{O}$ hydrogen bonds (Table 1 ).

\section{S2. Experimental}

$77 \%$ 3-Chloroperoxybenzoic acid (224 mg, $1.0 \mathrm{mmol})$ was added in small portions to a stirred solution of 5-cyclohexyl-2-(3-fluorophenyl)-3-methylsulfanyl-1-benzofuran (306 mg, $0.9 \mathrm{mmol})$ in dichloromethane $(30 \mathrm{~mL})$ at $273 \mathrm{~K}$. After being stirred at room temperature for $4 \mathrm{~h}$., the mixture was washed with saturated sodium bicarbonate solution and the organic layer was separated, dried over magnesium sulfate, filtered and concentrated at reduced pressure. The residue was purified by column chromatography (hexane-ethyl acetate, $1: 1 \mathrm{v} / \mathrm{v}$ ) to afford the title compound as a colorless solid [yield 76\%, m.p. 443-444 K; $R_{\mathrm{f}}=0.55$ (hexane-ethyl acetate, 1:1 v/v)]. Single crystals suitable for X-ray diffraction were prepared by slow evaporation of a solution of the title compound in acetone at room temperature.

\section{S3. Refinement}

All $\mathrm{H}$ atoms were positioned geometrically and refined using a riding model, with $\mathrm{C}-\mathrm{H}=0.95 \AA$ for aryl, $1.0 \AA$ for methine, $0.99 \AA$ for methylene and $0.98 \AA$ for methyl $\mathrm{H}$ atoms, respectively. $U_{\mathrm{iso}}(\mathrm{H})=1.2 U_{\mathrm{eq}}(\mathrm{C})$ for aryl, methine, and methylene, and $1.5 U_{\mathrm{eq}}(\mathrm{C})$ for methyl $\mathrm{H}$ atoms. 


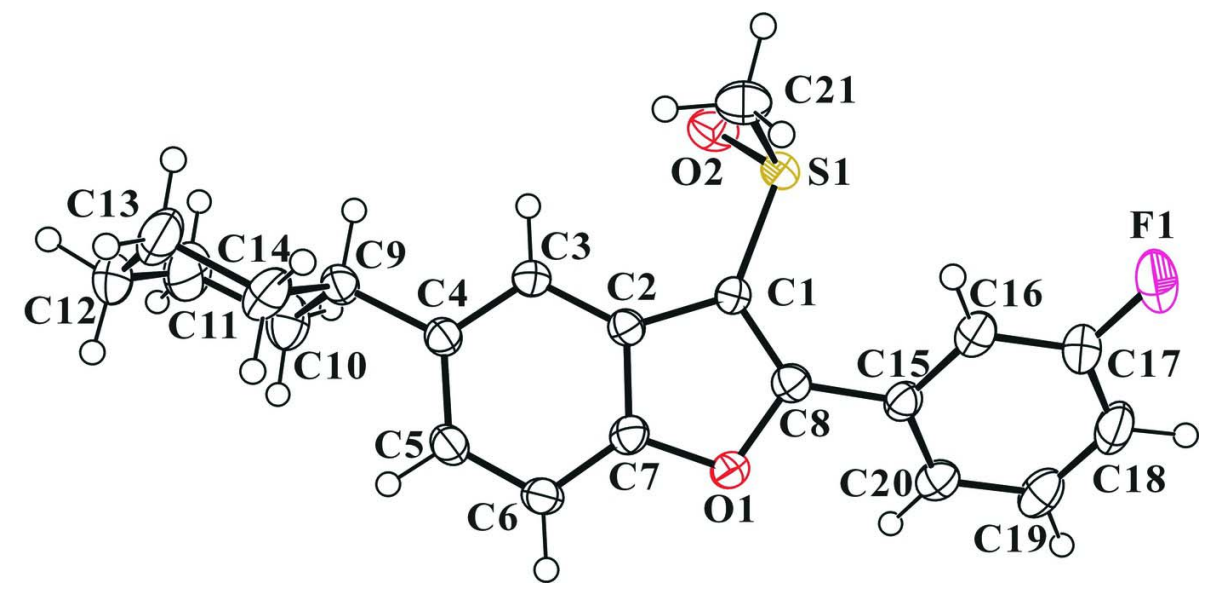

Figure 1

The molecular structure of the title compound with displacement ellipsoids drawn at the $50 \%$ probability level. $\mathrm{H}$ atoms are presented as small spheres of arbitrary radius.

5-Cyclohexyl-2-(3-fluorophenyl)-3-methylsulfinyl-1-benzofuran

Crystal data

$\mathrm{C}_{21} \mathrm{H}_{21} \mathrm{FO}_{2} \mathrm{~S}$

$M_{r}=356.44$

Orthorhombic, Pna2 ${ }_{1}$

Hall symbol: P 2c -2n

$a=12.7767$ (10) $\AA$

$b=13.0764$ (10) $\AA$

$c=10.7711(8) \AA$

$V=1799.6(2) \AA^{3}$

$Z=4$

$F(000)=752$

\section{Data collection}

\section{Bruker SMART APEXII CCD}

diffractometer

Radiation source: rotating anode

Graphite multilayer monochromator

Detector resolution: 10.0 pixels $\mathrm{mm}^{-1}$

$\varphi$ and $\omega$ scans

Absorption correction: multi-scan

(SADABS; Bruker, 2009)

$T_{\min }=0.935, T_{\max }=0.961$

\section{Refinement}

Refinement on $F^{2}$

Least-squares matrix: full

$R\left[F^{2}>2 \sigma\left(F^{2}\right)\right]=0.041$

$w R\left(F^{2}\right)=0.096$

$S=1.03$

4503 reflections

227 parameters

1 restraint

0 constraints
$D_{\mathrm{x}}=1.316 \mathrm{Mg} \mathrm{m}^{-3}$

Melting point: $443 \mathrm{~K}$

Mo $K \alpha$ radiation, $\lambda=0.71073 \AA$

Cell parameters from 4473 reflections

$\theta=2.5-28.1^{\circ}$

$\mu=0.20 \mathrm{~mm}^{-1}$

$T=173 \mathrm{~K}$

Block, colourless

$0.34 \times 0.24 \times 0.20 \mathrm{~mm}$

17893 measured reflections

4503 independent reflections

4036 reflections with $I>2 \sigma(I)$

$R_{\text {int }}=0.058$

$\theta_{\max }=28.4^{\circ}, \theta_{\min }=2.2^{\circ}$

$h=-17 \rightarrow 17$

$k=-17 \rightarrow 16$

$l=-14 \rightarrow 14$

Primary atom site location: structure-invariant direct methods

Secondary atom site location: difference Fourier map

Hydrogen site location: difference Fourier map

$\mathrm{H}$-atom parameters constrained

$w=1 /\left[\sigma^{2}\left(F_{\mathrm{o}}^{2}\right)+(0.0426 P)^{2}+0.2167 P\right]$

where $P=\left(F_{\mathrm{o}}^{2}+2 F_{\mathrm{c}}^{2}\right) / 3$

$(\Delta / \sigma)_{\max }<0.001$

$\Delta \rho_{\max }=0.33$ e $\AA^{-3}$ 
$\Delta \rho_{\min }=-0.25$ e $\AA^{-3}$
Absolute structure: Flack (1983), 2131 Friedel pairs

Absolute structure parameter: -0.07 (7)

Fractional atomic coordinates and isotropic or equivalent isotropic displacement parameters $\left(\hat{A}^{2}\right)$

\begin{tabular}{|c|c|c|c|c|}
\hline & $x$ & $y$ & $z$ & $U_{\text {iso }} * / U_{\text {eq }}$ \\
\hline S1 & $0.56575(3)$ & $0.02701(3)$ & $0.35040(5)$ & $0.02691(11)$ \\
\hline $\mathrm{F} 1$ & $0.77633(15)$ & $0.11534(12)$ & $-0.03264(18)$ & $0.0792(6)$ \\
\hline $\mathrm{O} 1$ & $0.46206(10)$ & $0.30803(10)$ & $0.29361(13)$ & $0.0277(3)$ \\
\hline $\mathrm{O} 2$ & $0.56405(11)$ & $-0.00184(12)$ & $0.48474(14)$ & 0.0390 (4) \\
\hline $\mathrm{C} 1$ & $0.49571(12)$ & $0.14257(13)$ & $0.33717(18)$ & $0.0234(3)$ \\
\hline $\mathrm{C} 2$ & $0.40393(13)$ & $0.17197(14)$ & $0.40656(17)$ & $0.0231(3)$ \\
\hline $\mathrm{C} 3$ & $0.33673(13)$ & $0.12486(14)$ & $0.49109(16)$ & 0.0254 (4) \\
\hline H3 & 0.3475 & 0.0555 & 0.5143 & $0.031 *$ \\
\hline $\mathrm{C} 4$ & $0.25446(14)$ & $0.17975(15)$ & $0.54083(17)$ & 0.0257 (4) \\
\hline $\mathrm{C} 5$ & $0.24014(14)$ & $0.28249(15)$ & $0.5060(2)$ & $0.0296(4)$ \\
\hline H5 & 0.1833 & 0.3196 & 0.5408 & $0.036^{*}$ \\
\hline C6 & $0.30589(14)$ & $0.33145(15)$ & $0.42275(19)$ & $0.0287(4)$ \\
\hline H6 & 0.2957 & 0.4009 & 0.3997 & $0.034^{*}$ \\
\hline $\mathrm{C} 7$ & $0.38672(13)$ & $0.27399(14)$ & $0.37531(16)$ & $0.0260(4)$ \\
\hline $\mathrm{C} 8$ & $0.52756(14)$ & $0.22666(14)$ & $0.27291(16)$ & $0.0256(4)$ \\
\hline C9 & $0.17818(14)$ & $0.12816(16)$ & $0.62864(17)$ & $0.0287(4)$ \\
\hline H9 & 0.2064 & 0.0585 & 0.6469 & $0.034 *$ \\
\hline $\mathrm{C} 10$ & $0.16664(19)$ & $0.1834(2)$ & $0.7521(2)$ & $0.0443(5)$ \\
\hline $\mathrm{H} 10 \mathrm{~A}$ & 0.1439 & 0.2547 & 0.7367 & $0.053^{*}$ \\
\hline H10B & 0.2354 & 0.1858 & 0.7943 & $0.053^{*}$ \\
\hline $\mathrm{C} 11$ & $0.08727(19)$ & $0.1306(2)$ & $0.8365(2)$ & $0.0554(7)$ \\
\hline H11A & 0.1153 & 0.0634 & 0.8625 & $0.067^{*}$ \\
\hline H11B & 0.0767 & 0.1725 & 0.9121 & $0.067^{*}$ \\
\hline C12 & $-0.01698(19)$ & $0.1153(2)$ & $0.7722(3)$ & $0.0573(8)$ \\
\hline $\mathrm{H} 12 \mathrm{~A}$ & -0.0642 & 0.0761 & 0.8273 & $0.069^{*}$ \\
\hline H12B & -0.0495 & 0.1827 & 0.7564 & $0.069^{*}$ \\
\hline C13 & $-0.00474(18)$ & $0.0591(2)$ & $0.6512(3)$ & $0.0494(6)$ \\
\hline $\mathrm{H} 13 \mathrm{~A}$ & -0.0736 & 0.0540 & 0.6095 & $0.059 *$ \\
\hline H13B & 0.0206 & -0.0112 & 0.6676 & $0.059^{*}$ \\
\hline C14 & $0.07219(16)$ & $0.1139(2)$ & $0.5661(2)$ & $0.0405(5)$ \\
\hline H14A & 0.0434 & 0.1816 & 0.5431 & $0.049 *$ \\
\hline H14B & 0.0813 & 0.0737 & 0.4890 & $0.049^{*}$ \\
\hline C15 & $0.61838(13)$ & $0.24653(16)$ & $0.19341(17)$ & $0.0269(4)$ \\
\hline $\mathrm{C} 16$ & $0.65392(17)$ & $0.17134(18)$ & $0.1135(2)$ & $0.0389(5)$ \\
\hline H16 & 0.6172 & 0.1086 & 0.1051 & $0.047^{*}$ \\
\hline $\mathrm{C} 17$ & $0.74380(18)$ & $0.18950(19)$ & $0.0462(2)$ & $0.0457(6)$ \\
\hline $\mathrm{C} 18$ & $0.79812(17)$ & 0.27964 (19) & $0.0536(2)$ & $0.0421(5)$ \\
\hline H18 & 0.8604 & 0.2896 & 0.0069 & $0.051^{*}$ \\
\hline C19 & $0.76030(16)$ & $0.35559(19)$ & $0.1303(2)$ & $0.0376(5)$ \\
\hline H19 & 0.7957 & 0.4194 & 0.1349 & $0.045^{*}$ \\
\hline $\mathrm{C} 20$ & $0.67083(14)$ & $0.33938(16)$ & $0.20081(19)$ & $0.0306(4)$ \\
\hline
\end{tabular}




$\begin{array}{lllll}\text { H20 } & 0.6455 & 0.3917 & 0.2541 & 0.037^{*} \\ \text { C21 } & 0.47422(18) & -0.05631(16) & 0.2771(2) & 0.0378(5) \\ \text { H21A } & 0.5009 & -0.1266 & 0.2794 & 0.057^{*} \\ \text { H21B } & 0.4644 & -0.0352 & 0.1905 & 0.057^{*} \\ \text { H21C } & 0.4071 & -0.0530 & 0.3209 & 0.057^{*}\end{array}$

Atomic displacement parameters $\left(\AA^{2}\right)$

\begin{tabular}{lllllll}
\hline & $U^{11}$ & $U^{22}$ & $U^{33}$ & $U^{12}$ & $U^{13}$ & $U^{23}$ \\
\hline S1 & $0.02477(19)$ & $0.0252(2)$ & $0.0307(2)$ & $0.00363(16)$ & $0.00124(19)$ & $0.0016(2)$ \\
F1 & $0.0930(12)$ & $0.0564(10)$ & $0.0883(13)$ & $0.0007(9)$ & $0.0641(10)$ & $-0.0086(9)$ \\
O1 & $0.0257(6)$ & $0.0243(7)$ & $0.0332(7)$ & $-0.0006(5)$ & $0.0024(5)$ & $0.0051(6)$ \\
O2 & $0.0457(9)$ & $0.0377(8)$ & $0.0335(8)$ & $0.0094(7)$ & $-0.0076(7)$ & $0.0065(7)$ \\
C1 & $0.0219(7)$ & $0.0237(8)$ & $0.0246(9)$ & $-0.0008(6)$ & $0.0011(7)$ & $0.0004(8)$ \\
C2 & $0.0207(7)$ & $0.0243(9)$ & $0.0243(8)$ & $0.0011(7)$ & $-0.0022(6)$ & $0.0010(7)$ \\
C3 & $0.0255(8)$ & $0.0236(9)$ & $0.0272(9)$ & $0.0014(7)$ & $0.0001(7)$ & $0.0023(7)$ \\
C4 & $0.0246(9)$ & $0.0267(10)$ & $0.0256(9)$ & $0.0020(7)$ & $0.0008(7)$ & $0.0007(7)$ \\
C5 & $0.0248(9)$ & $0.0291(10)$ & $0.0350(10)$ & $0.0042(7)$ & $0.0020(7)$ & $-0.0021(8)$ \\
C6 & $0.0279(9)$ & $0.0232(9)$ & $0.0352(10)$ & $0.0026(7)$ & $-0.0013(8)$ & $0.0013(8)$ \\
C7 & $0.0219(8)$ & $0.0262(9)$ & $0.0301(10)$ & $-0.0030(7)$ & $-0.0022(7)$ & $0.0023(7)$ \\
C8 & $0.0244(8)$ & $0.0264(10)$ & $0.0260(9)$ & $0.0005(7)$ & $-0.0019(7)$ & $0.0011(7)$ \\
C9 & $0.0284(9)$ & $0.0306(10)$ & $0.0271(9)$ & $0.0055(7)$ & $0.0042(7)$ & $0.0033(8)$ \\
C10 & $0.0440(12)$ & $0.0575(15)$ & $0.0315(11)$ & $-0.0056(11)$ & $0.0084(9)$ & $-0.0057(10)$ \\
C11 & $0.0643(15)$ & $0.0647(17)$ & $0.0373(13)$ & $-0.0022(12)$ & $0.0242(12)$ & $-0.0026(13)$ \\
C12 & $0.0434(13)$ & $0.0520(15)$ & $0.077(2)$ & $0.0069(12)$ & $0.0309(13)$ & $0.0186(14)$ \\
C13 & $0.0353(11)$ & $0.0559(16)$ & $0.0571(15)$ & $-0.0109(11)$ & $0.0033(10)$ & $0.0208(12)$ \\
C14 & $0.0383(12)$ & $0.0457(14)$ & $0.0376(11)$ & $-0.0115(10)$ & $-0.0014(9)$ & $0.0124(10)$ \\
C15 & $0.0250(8)$ & $0.0297(10)$ & $0.0261(9)$ & $0.0002(7)$ & $0.0008(7)$ & $0.0066(7)$ \\
C16 & $0.0445(12)$ & $0.0335(12)$ & $0.0387(12)$ & $-0.0035(9)$ & $0.0140(9)$ & $0.0040(9)$ \\
C17 & $0.0474(13)$ & $0.0440(14)$ & $0.0457(13)$ & $0.0073(11)$ & $0.0228(11)$ & $0.0087(11)$ \\
C18 & $0.0297(10)$ & $0.0564(15)$ & $0.0402(12)$ & $0.0000(10)$ & $0.0101(9)$ & $0.0163(11)$ \\
C19 & $0.0295(10)$ & $0.0448(13)$ & $0.0386(11)$ & $-0.0125(9)$ & $-0.0027(9)$ & $0.0095(10)$ \\
C20 & $0.0289(9)$ & $0.0315(11)$ & $0.0312(10)$ & $-0.0024(8)$ & $-0.0044(8)$ & $0.0049(8)$ \\
C21 & $0.0447(12)$ & $0.0281(10)$ & $0.0406(12)$ & $-0.0025(9)$ & $-0.0070(9)$ & $-0.0039(9)$ \\
& & & & & & \\
\hline & & & & & & \\
\hline
\end{tabular}

Geometric parameters $\left(A,{ }^{\circ}\right)$

\begin{tabular}{llll}
\hline $\mathrm{S} 1-\mathrm{O} 2$ & $1.4954(15)$ & $\mathrm{C} 11-\mathrm{C} 12$ & $1.515(4)$ \\
$\mathrm{S} 1-\mathrm{C} 1$ & $1.7620(17)$ & $\mathrm{C} 11-\mathrm{H} 11 \mathrm{~A}$ & 0.9900 \\
$\mathrm{~S} 1-\mathrm{C} 21$ & $1.783(2)$ & $\mathrm{C} 11-\mathrm{H} 11 \mathrm{~B}$ & 0.9900 \\
$\mathrm{~F} 1-\mathrm{C} 17$ & $1.355(3)$ & $\mathrm{C} 12-\mathrm{C} 13$ & $1.505(4)$ \\
$\mathrm{O} 1-\mathrm{C} 8$ & $1.372(2)$ & $\mathrm{C} 12-\mathrm{H} 12 \mathrm{~A}$ & 0.9900 \\
$\mathrm{O} 1-\mathrm{C} 7$ & $1.378(2)$ & $\mathrm{C} 12-\mathrm{H} 12 \mathrm{~B}$ & 0.9900 \\
$\mathrm{C} 1-\mathrm{C} 8$ & $1.361(3)$ & $\mathrm{C} 13-\mathrm{C} 14$ & $1.523(3)$ \\
$\mathrm{C} 1-\mathrm{C} 2$ & $1.443(2)$ & $\mathrm{C} 13-\mathrm{H} 13 \mathrm{~A}$ & 0.9900 \\
$\mathrm{C} 2-\mathrm{C} 7$ & $1.393(3)$ & $\mathrm{C} 13-\mathrm{H} 13 \mathrm{~B}$ & 0.9900 \\
$\mathrm{C} 2-\mathrm{C} 3$ & $1.395(2)$ & $\mathrm{C} 14-\mathrm{H} 14 \mathrm{~A}$ & 0.9900 \\
$\mathrm{C} 3-\mathrm{C} 4$ & $1.381(2)$ & $\mathrm{C} 14-\mathrm{H} 14 \mathrm{~B}$ & 0.9900
\end{tabular}




\begin{tabular}{|c|c|c|c|}
\hline $\mathrm{C} 3-\mathrm{H} 3$ & 0.9500 & $\mathrm{C} 15-\mathrm{C} 16$ & $1.383(3)$ \\
\hline $\mathrm{C} 4-\mathrm{C} 5$ & $1.407(3)$ & $\mathrm{C} 15-\mathrm{C} 20$ & $1.389(3)$ \\
\hline $\mathrm{C} 4-\mathrm{C} 9$ & $1.516(3)$ & $\mathrm{C} 16-\mathrm{C} 17$ & $1.378(3)$ \\
\hline $\mathrm{C} 5-\mathrm{C} 6$ & $1.385(3)$ & $\mathrm{C} 16-\mathrm{H} 16$ & 0.9500 \\
\hline $\mathrm{C} 5-\mathrm{H} 5$ & 0.9500 & $\mathrm{C} 17-\mathrm{C} 18$ & $1.370(3)$ \\
\hline $\mathrm{C} 6-\mathrm{C} 7$ & $1.376(3)$ & $\mathrm{C} 18-\mathrm{C} 19$ & $1.379(3)$ \\
\hline C6-H6 & 0.9500 & C18-H18 & 0.9500 \\
\hline $\mathrm{C} 8-\mathrm{C} 15$ & $1.465(2)$ & $\mathrm{C} 19-\mathrm{C} 20$ & $1.389(3)$ \\
\hline $\mathrm{C} 9-\mathrm{C} 10$ & $1.520(3)$ & $\mathrm{C} 19-\mathrm{H} 19$ & 0.9500 \\
\hline $\mathrm{C} 9-\mathrm{C} 14$ & $1.524(3)$ & $\mathrm{C} 20-\mathrm{H} 20$ & 0.9500 \\
\hline C9- $\mathrm{H} 9$ & 1.0000 & $\mathrm{C} 21-\mathrm{H} 21 \mathrm{~A}$ & 0.9800 \\
\hline $\mathrm{C} 10-\mathrm{C} 11$ & $1.527(3)$ & $\mathrm{C} 21-\mathrm{H} 21 \mathrm{~B}$ & 0.9800 \\
\hline $\mathrm{C} 10-\mathrm{H} 10 \mathrm{~A}$ & 0.9900 & $\mathrm{C} 21-\mathrm{H} 21 \mathrm{C}$ & 0.9800 \\
\hline $\mathrm{C} 10-\mathrm{H} 10 \mathrm{~B}$ & 0.9900 & & \\
\hline $\mathrm{O} 2-\mathrm{S} 1-\mathrm{C} 1$ & $106.69(9)$ & $\mathrm{C} 10-\mathrm{C} 11-\mathrm{H} 11 \mathrm{~B}$ & 109.3 \\
\hline $\mathrm{O} 2-\mathrm{S} 1-\mathrm{C} 21$ & $105.37(10)$ & $\mathrm{H} 11 \mathrm{~A}-\mathrm{C} 11-\mathrm{H} 11 \mathrm{~B}$ & 107.9 \\
\hline $\mathrm{C} 1-\mathrm{S} 1-\mathrm{C} 21$ & $98.93(9)$ & $\mathrm{C} 13-\mathrm{C} 12-\mathrm{C} 11$ & $111.69(19)$ \\
\hline $\mathrm{C} 8-\mathrm{O} 1-\mathrm{C} 7$ & $106.23(14)$ & $\mathrm{C} 13-\mathrm{C} 12-\mathrm{H} 12 \mathrm{~A}$ & 109.3 \\
\hline $\mathrm{C} 8-\mathrm{C} 1-\mathrm{C} 2$ & $106.92(15)$ & $\mathrm{C} 11-\mathrm{C} 12-\mathrm{H} 12 \mathrm{~A}$ & 109.3 \\
\hline $\mathrm{C} 8-\mathrm{C} 1-\mathrm{S} 1$ & $125.59(13)$ & $\mathrm{C} 13-\mathrm{C} 12-\mathrm{H} 12 \mathrm{~B}$ & 109.3 \\
\hline $\mathrm{C} 2-\mathrm{C} 1-\mathrm{S} 1$ & $126.83(13)$ & $\mathrm{C} 11-\mathrm{C} 12-\mathrm{H} 12 \mathrm{~B}$ & 109.3 \\
\hline $\mathrm{C} 7-\mathrm{C} 2-\mathrm{C} 3$ & $118.91(16)$ & $\mathrm{H} 12 \mathrm{~A}-\mathrm{C} 12-\mathrm{H} 12 \mathrm{~B}$ & 107.9 \\
\hline $\mathrm{C} 7-\mathrm{C} 2-\mathrm{C} 1$ & $104.97(15)$ & $\mathrm{C} 12-\mathrm{C} 13-\mathrm{C} 14$ & $111.0(2)$ \\
\hline $\mathrm{C} 3-\mathrm{C} 2-\mathrm{C} 1$ & $136.11(17)$ & $\mathrm{C} 12-\mathrm{C} 13-\mathrm{H} 13 \mathrm{~A}$ & 109.4 \\
\hline $\mathrm{C} 4-\mathrm{C} 3-\mathrm{C} 2$ & $119.48(17)$ & $\mathrm{C} 14-\mathrm{C} 13-\mathrm{H} 13 \mathrm{~A}$ & 109.4 \\
\hline $\mathrm{C} 4-\mathrm{C} 3-\mathrm{H} 3$ & 120.3 & $\mathrm{C} 12-\mathrm{C} 13-\mathrm{H} 13 \mathrm{~B}$ & 109.4 \\
\hline $\mathrm{C} 2-\mathrm{C} 3-\mathrm{H} 3$ & 120.3 & $\mathrm{C} 14-\mathrm{C} 13-\mathrm{H} 13 \mathrm{~B}$ & 109.4 \\
\hline $\mathrm{C} 3-\mathrm{C} 4-\mathrm{C} 5$ & $119.46(17)$ & $\mathrm{H} 13 \mathrm{~A}-\mathrm{C} 13-\mathrm{H} 13 \mathrm{~B}$ & 108.0 \\
\hline $\mathrm{C} 3-\mathrm{C} 4-\mathrm{C} 9$ & $120.00(17)$ & $\mathrm{C} 13-\mathrm{C} 14-\mathrm{C} 9$ & $111.42(18)$ \\
\hline $\mathrm{C} 5-\mathrm{C} 4-\mathrm{C} 9$ & $120.51(16)$ & $\mathrm{C} 13-\mathrm{C} 14-\mathrm{H} 14 \mathrm{~A}$ & 109.3 \\
\hline $\mathrm{C} 6-\mathrm{C} 5-\mathrm{C} 4$ & $122.35(17)$ & $\mathrm{C} 9-\mathrm{C} 14-\mathrm{H} 14 \mathrm{~A}$ & 109.3 \\
\hline $\mathrm{C} 6-\mathrm{C} 5-\mathrm{H} 5$ & 118.8 & $\mathrm{C} 13-\mathrm{C} 14-\mathrm{H} 14 \mathrm{~B}$ & 109.3 \\
\hline $\mathrm{C} 4-\mathrm{C} 5-\mathrm{H} 5$ & 118.8 & C9-C14-H14B & 109.3 \\
\hline $\mathrm{C} 7-\mathrm{C} 6-\mathrm{C} 5$ & $116.31(17)$ & $\mathrm{H} 14 \mathrm{~A}-\mathrm{C} 14-\mathrm{H} 14 \mathrm{~B}$ & 108.0 \\
\hline $\mathrm{C} 7-\mathrm{C} 6-\mathrm{H} 6$ & 121.8 & $\mathrm{C} 16-\mathrm{C} 15-\mathrm{C} 20$ & $119.90(18)$ \\
\hline $\mathrm{C} 5-\mathrm{C} 6-\mathrm{H} 6$ & 121.8 & $\mathrm{C} 16-\mathrm{C} 15-\mathrm{C} 8$ & $119.84(18)$ \\
\hline $\mathrm{C} 6-\mathrm{C} 7-\mathrm{O} 1$ & $125.82(17)$ & $\mathrm{C} 20-\mathrm{C} 15-\mathrm{C} 8$ & $120.22(18)$ \\
\hline $\mathrm{C} 6-\mathrm{C} 7-\mathrm{C} 2$ & $123.48(17)$ & $\mathrm{C} 17-\mathrm{C} 16-\mathrm{C} 15$ & $118.6(2)$ \\
\hline $\mathrm{O} 1-\mathrm{C} 7-\mathrm{C} 2$ & $110.69(15)$ & $\mathrm{C} 17-\mathrm{C} 16-\mathrm{H} 16$ & 120.7 \\
\hline $\mathrm{C} 1-\mathrm{C} 8-\mathrm{O} 1$ & $111.19(15)$ & $\mathrm{C} 15-\mathrm{C} 16-\mathrm{H} 16$ & 120.7 \\
\hline $\mathrm{C} 1-\mathrm{C} 8-\mathrm{C} 15$ & $132.60(17)$ & $\mathrm{F} 1-\mathrm{C} 17-\mathrm{C} 18$ & $119.79(19)$ \\
\hline $\mathrm{O} 1-\mathrm{C} 8-\mathrm{C} 15$ & $116.14(16)$ & $\mathrm{F} 1-\mathrm{C} 17-\mathrm{C} 16$ & $117.5(2)$ \\
\hline $\mathrm{C} 4-\mathrm{C} 9-\mathrm{C} 10$ & $113.34(18)$ & $\mathrm{C} 18-\mathrm{C} 17-\mathrm{C} 16$ & $122.7(2)$ \\
\hline $\mathrm{C} 4-\mathrm{C} 9-\mathrm{C} 14$ & $110.48(16)$ & $\mathrm{C} 17-\mathrm{C} 18-\mathrm{C} 19$ & $118.5(2)$ \\
\hline $\mathrm{C} 10-\mathrm{C} 9-\mathrm{C} 14$ & $111.02(18)$ & $\mathrm{C} 17-\mathrm{C} 18-\mathrm{H} 18$ & 120.8 \\
\hline $\mathrm{C} 4-\mathrm{C} 9-\mathrm{H} 9$ & 107.2 & $\mathrm{C} 19-\mathrm{C} 18-\mathrm{H} 18$ & 120.8 \\
\hline $\mathrm{C} 10-\mathrm{C} 9-\mathrm{H} 9$ & 107.2 & $\mathrm{C} 18-\mathrm{C} 19-\mathrm{C} 20$ & $120.4(2)$ \\
\hline
\end{tabular}




\begin{tabular}{|c|c|c|c|}
\hline $\mathrm{C} 14-\mathrm{C} 9-\mathrm{H} 9$ & 107.2 & $\mathrm{C} 18-\mathrm{C} 19-\mathrm{H} 19$ & 119.8 \\
\hline $\mathrm{C} 9-\mathrm{C} 10-\mathrm{C} 11$ & $111.7(2)$ & $\mathrm{C} 20-\mathrm{C} 19-\mathrm{H} 19$ & 119.8 \\
\hline $\mathrm{C} 9-\mathrm{C} 10-\mathrm{H} 10 \mathrm{~A}$ & 109.3 & $\mathrm{C} 19-\mathrm{C} 20-\mathrm{C} 15$ & $119.9(2)$ \\
\hline $\mathrm{C} 11-\mathrm{C} 10-\mathrm{H} 10 \mathrm{~A}$ & 109.3 & $\mathrm{C} 19-\mathrm{C} 20-\mathrm{H} 20$ & 120.0 \\
\hline $\mathrm{C} 9-\mathrm{C} 10-\mathrm{H} 10 \mathrm{~B}$ & 109.3 & $\mathrm{C} 15-\mathrm{C} 20-\mathrm{H} 20$ & 120.0 \\
\hline $\mathrm{C} 11-\mathrm{C} 10-\mathrm{H} 10 \mathrm{~B}$ & 109.3 & $\mathrm{~S} 1-\mathrm{C} 21-\mathrm{H} 21 \mathrm{~A}$ & 109.5 \\
\hline $\mathrm{H} 10 \mathrm{~A}-\mathrm{C} 10-\mathrm{H} 10 \mathrm{~B}$ & 107.9 & $\mathrm{~S} 1-\mathrm{C} 21-\mathrm{H} 21 \mathrm{~B}$ & 109.5 \\
\hline $\mathrm{C} 12-\mathrm{C} 11-\mathrm{C} 10$ & $111.8(2)$ & $\mathrm{H} 21 \mathrm{~A}-\mathrm{C} 21-\mathrm{H} 21 \mathrm{~B}$ & 109.5 \\
\hline $\mathrm{C} 12-\mathrm{C} 11-\mathrm{H} 11 \mathrm{~A}$ & 109.3 & $\mathrm{~S} 1-\mathrm{C} 21-\mathrm{H} 21 \mathrm{C}$ & 109.5 \\
\hline $\mathrm{C} 10-\mathrm{C} 11-\mathrm{H} 11 \mathrm{~A}$ & 109.3 & $\mathrm{H} 21 \mathrm{~A}-\mathrm{C} 21-\mathrm{H} 21 \mathrm{C}$ & 109.5 \\
\hline $\mathrm{C} 12-\mathrm{C} 11-\mathrm{H} 11 \mathrm{~B}$ & 109.3 & $\mathrm{H} 21 \mathrm{~B}-\mathrm{C} 21-\mathrm{H} 21 \mathrm{C}$ & 109.5 \\
\hline $\mathrm{O} 2-\mathrm{S} 1-\mathrm{C} 1-\mathrm{C} 8$ & $133.15(16)$ & $\mathrm{C} 7-\mathrm{O} 1-\mathrm{C} 8-\mathrm{C} 15$ & $-176.61(15)$ \\
\hline $\mathrm{C} 21-\mathrm{S} 1-\mathrm{C} 1-\mathrm{C} 8$ & $-117.75(17)$ & $\mathrm{C} 3-\mathrm{C} 4-\mathrm{C} 9-\mathrm{C} 10$ & $125.5(2)$ \\
\hline $\mathrm{O} 2-\mathrm{S} 1-\mathrm{C} 1-\mathrm{C} 2$ & $-36.29(18)$ & $\mathrm{C} 5-\mathrm{C} 4-\mathrm{C} 9-\mathrm{C} 10$ & $-56.7(2)$ \\
\hline $\mathrm{C} 21-\mathrm{S} 1-\mathrm{C} 1-\mathrm{C} 2$ & $72.81(18)$ & $\mathrm{C} 3-\mathrm{C} 4-\mathrm{C} 9-\mathrm{C} 14$ & $-109.2(2)$ \\
\hline $\mathrm{C} 8-\mathrm{C} 1-\mathrm{C} 2-\mathrm{C} 7$ & $1.11(19)$ & $\mathrm{C} 5-\mathrm{C} 4-\mathrm{C} 9-\mathrm{C} 14$ & $68.6(2)$ \\
\hline $\mathrm{S} 1-\mathrm{C} 1-\mathrm{C} 2-\mathrm{C} 7$ & $172.15(13)$ & $\mathrm{C} 4-\mathrm{C} 9-\mathrm{C} 10-\mathrm{C} 11$ & $178.61(19)$ \\
\hline $\mathrm{C} 8-\mathrm{C} 1-\mathrm{C} 2-\mathrm{C} 3$ & $-177.8(2)$ & $\mathrm{C} 14-\mathrm{C} 9-\mathrm{C} 10-\mathrm{C} 11$ & $53.6(3)$ \\
\hline $\mathrm{S} 1-\mathrm{C} 1-\mathrm{C} 2-\mathrm{C} 3$ & $-6.8(3)$ & $\mathrm{C} 9-\mathrm{C} 10-\mathrm{C} 11-\mathrm{C} 12$ & $-53.3(3)$ \\
\hline $\mathrm{C} 7-\mathrm{C} 2-\mathrm{C} 3-\mathrm{C} 4$ & $0.6(3)$ & $\mathrm{C} 10-\mathrm{C} 11-\mathrm{C} 12-\mathrm{C} 13$ & $54.5(3)$ \\
\hline $\mathrm{C} 1-\mathrm{C} 2-\mathrm{C} 3-\mathrm{C} 4$ & $179.4(2)$ & $\mathrm{C} 11-\mathrm{C} 12-\mathrm{C} 13-\mathrm{C} 14$ & $-55.9(3)$ \\
\hline $\mathrm{C} 2-\mathrm{C} 3-\mathrm{C} 4-\mathrm{C} 5$ & $-0.4(3)$ & $\mathrm{C} 12-\mathrm{C} 13-\mathrm{C} 14-\mathrm{C} 9$ & $56.4(3)$ \\
\hline $\mathrm{C} 2-\mathrm{C} 3-\mathrm{C} 4-\mathrm{C} 9$ & $177.44(16)$ & $\mathrm{C} 4-\mathrm{C} 9-\mathrm{C} 14-\mathrm{C} 13$ & $178.2(2)$ \\
\hline $\mathrm{C} 3-\mathrm{C} 4-\mathrm{C} 5-\mathrm{C} 6$ & $0.0(3)$ & $\mathrm{C} 10-\mathrm{C} 9-\mathrm{C} 14-\mathrm{C} 13$ & $-55.2(3)$ \\
\hline $\mathrm{C} 9-\mathrm{C} 4-\mathrm{C} 5-\mathrm{C} 6$ & $-177.75(18)$ & $\mathrm{C} 1-\mathrm{C} 8-\mathrm{C} 15-\mathrm{C} 16$ & $39.0(3)$ \\
\hline $\mathrm{C} 4-\mathrm{C} 5-\mathrm{C} 6-\mathrm{C} 7$ & $0.0(3)$ & $\mathrm{O} 1-\mathrm{C} 8-\mathrm{C} 15-\mathrm{C} 16$ & $-144.42(19)$ \\
\hline $\mathrm{C} 5-\mathrm{C} 6-\mathrm{C} 7-\mathrm{O} 1$ & $-178.59(17)$ & $\mathrm{C} 1-\mathrm{C} 8-\mathrm{C} 15-\mathrm{C} 20$ & $-139.0(2)$ \\
\hline $\mathrm{C} 5-\mathrm{C} 6-\mathrm{C} 7-\mathrm{C} 2$ & $0.2(3)$ & $\mathrm{O} 1-\mathrm{C} 8-\mathrm{C} 15-\mathrm{C} 20$ & $37.6(2)$ \\
\hline $\mathrm{C} 8-\mathrm{O} 1-\mathrm{C} 7-\mathrm{C} 6$ & $178.97(17)$ & $\mathrm{C} 20-\mathrm{C} 15-\mathrm{C} 16-\mathrm{C} 17$ & $2.5(3)$ \\
\hline $\mathrm{C} 8-\mathrm{O} 1-\mathrm{C} 7-\mathrm{C} 2$ & $0.04(19)$ & $\mathrm{C} 8-\mathrm{C} 15-\mathrm{C} 16-\mathrm{C} 17$ & $-175.5(2)$ \\
\hline $\mathrm{C} 3-\mathrm{C} 2-\mathrm{C} 7-\mathrm{C} 6$ & $-0.5(3)$ & $\mathrm{C} 15-\mathrm{C} 16-\mathrm{C} 17-\mathrm{F} 1$ & $-179.1(2)$ \\
\hline $\mathrm{C} 1-\mathrm{C} 2-\mathrm{C} 7-\mathrm{C} 6$ & $-179.67(17)$ & $\mathrm{C} 15-\mathrm{C} 16-\mathrm{C} 17-\mathrm{C} 18$ & $-1.3(4)$ \\
\hline $\mathrm{C} 3-\mathrm{C} 2-\mathrm{C} 7-\mathrm{O} 1$ & $178.43(15)$ & $\mathrm{F} 1-\mathrm{C} 17-\mathrm{C} 18-\mathrm{C} 19$ & $176.9(2)$ \\
\hline $\mathrm{C} 1-\mathrm{C} 2-\mathrm{C} 7-\mathrm{O} 1$ & $-0.7(2)$ & $\mathrm{C} 16-\mathrm{C} 17-\mathrm{C} 18-\mathrm{C} 19$ & $-0.9(4)$ \\
\hline $\mathrm{C} 2-\mathrm{C} 1-\mathrm{C} 8-\mathrm{O} 1$ & $-1.1(2)$ & $\mathrm{C} 17-\mathrm{C} 18-\mathrm{C} 19-\mathrm{C} 20$ & $1.8(3)$ \\
\hline $\mathrm{S} 1-\mathrm{C} 1-\mathrm{C} 8-\mathrm{O} 1$ & $-172.33(13)$ & $\mathrm{C} 18-\mathrm{C} 19-\mathrm{C} 20-\mathrm{C} 15$ & $-0.6(3)$ \\
\hline $\mathrm{C} 2-\mathrm{C} 1-\mathrm{C} 8-\mathrm{C} 15$ & $175.59(19)$ & $\mathrm{C} 16-\mathrm{C} 15-\mathrm{C} 20-\mathrm{C} 19$ & $-1.6(3)$ \\
\hline $\mathrm{S} 1-\mathrm{C} 1-\mathrm{C} 8-\mathrm{C} 15$ & $4.4(3)$ & $\mathrm{C} 8-\mathrm{C} 15-\mathrm{C} 20-\mathrm{C} 19$ & $176.39(18)$ \\
\hline $\mathrm{C} 7-\mathrm{O} 1-\mathrm{C} 8-\mathrm{C} 1$ & $0.71(19)$ & & \\
\hline
\end{tabular}

Hydrogen-bond geometry $\left(\AA,{ }^{\circ}\right)$

\begin{tabular}{lllll}
\hline$D-\mathrm{H} \cdots A$ & $D-\mathrm{H}$ & $\mathrm{H} \cdots A$ & $D \cdots A$ & $D-\mathrm{H} \cdots A$ \\
\hline $\mathrm{C} 21-\mathrm{H} 21 B \cdots \mathrm{O} 2^{\mathrm{i}}$ & 0.98 & 2.30 & $3.276(3)$ & 176 \\
\hline
\end{tabular}

Symmetry code: (i) $-x+1,-y, z-1 / 2$. 